\title{
Detection of His-Purkinje Activity from Distant Intracardiac Electrograms
}

\author{
LA Borowitz ${ }^{1}$, W Combs $^{2}$, EJ Berbari ${ }^{1}$ \\ ${ }^{1}$ Indiana University Purdue University Indianapolis, Indianapolis, IN, USA \\ ${ }^{2}$ Medtronic, Inc, Minneapolis, MN, USA
}

\begin{abstract}
The aim of this study was to determine the feasibility of detecting His-Purkinje system (HPS) activity from an intracardiac lead field (HR bipole) between catheter electrodes in the high right atrium and the right ventricular apex. A secondary endpoint was to determine if the resolution and sampling typically used in pacemakers would reproduce the electrophysiology (EP) lab results. Using data obtained during EP studies, HR bipole recordings were signal averaged and bandpass filtered. The interval between the onset of HPS activity and the onset of the QRS complex (HV interval) in the HR bipole was measured in 7 datasets and compared to His Bundle Electrograms (HBE). The HR bipole and distal $H B E$ produced linearly related results, offset by the delay of $18 \mathrm{msec}$ attributed to the time between actual HPS onset and the HPS onset in the distal HBE recording. A/D resolution was degraded and data were down sampled to $244 \mathrm{~Hz}$ and 8 bits, respectively to simulate pacemaker recordings. There was little to no detectable HPS activity in these pacemaker-simulated recordings. Additional data is necessary before conclusions can be made about the effectiveness of the HR bipole for HV measurement with an implantable device using these reduced data acquisition parameters.
\end{abstract}

\section{Introduction}

This study aims to determine the feasibility of detecting His-Purkinje System (HPS) activity from unipolar catheters placed in the high right atrium (HRA) and the right ventricular apex (RVA). The placement of electrodes is based on that of a dual chamber pacemaker with one electrode in the HRA and the other in the RVA. Current pacemakers sense from the HRA, RVA, or both, but they do not combine the two positions to create a wide lead field. Detecting His-Purkinje activity is not part of a pacemaker's sensing protocol. Currently, HPS activity is only measurable from catheters in very close proximity to these tissues (His Bundle ElectrogramsHBE) or on the body surface using signal averaging to improve the signal to noise ratio (SNR) of these very small signals. [1]
Figure 1 illustrates the lead field created by the HR bipole between the HRA and RVA unipolar tip electrodes. The HRA is the upper left pole of the field and the RVA is the lower right pole. The HR bipole contains the common signals between the unipolar HRA and RVA recordings. The HR bipole is a lead field that extends out in all dimensions from the two poles.

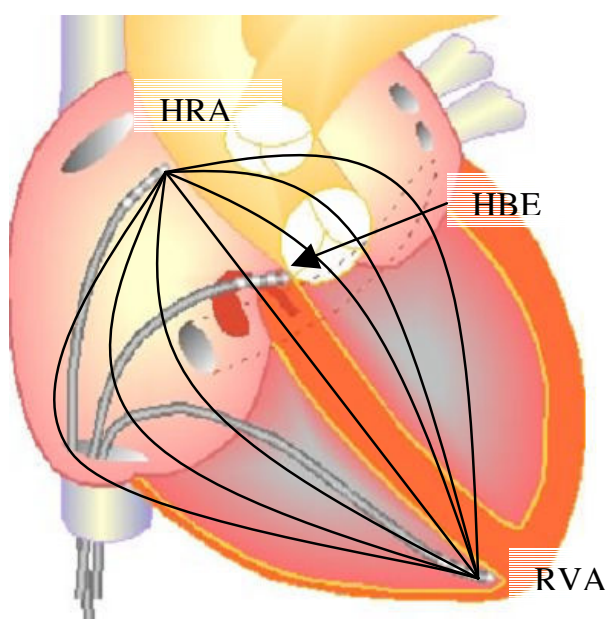

Figure 1: Partial view of the lead field created between HRA and RVA unipolar recordings. This field encompasses the HPS. (Courtesy of John Miller, M.D.)

\section{Methods}

Approval was granted by the Institutional Review Board of Indiana University (IRB) for this study. Data were retrieved from a General Electric Medical Systems (GEMS) Prucka CardioLab Electrophysiology (EP) system database including electrograms sampled at $1000 \mathrm{~Hz}$ with 12-bit A/D conversion. Leads selected were unipolar unfiltered HRA and RVA. Filtered bipolar HBE (proximal, medial and distal) were also selected for confirmation of HPS detection.

\subsection{Detection of the Onset of QRS Complex}

The threshold detection consisted of two requirements. The first was to have the signal amplitude greater than $2 / 3$ the maximum amplitude of the surface 
lead I. This will avoid mistaking the $\mathrm{P}$ wave for a QRS complex. The second requirement was to have the time derivative greater than $30 \mu \mathrm{V} / \mathrm{msec}$.

Surface leads I, II and II were combined to create the surface vector lead. A time frame of $20 \mathrm{msec}$ before the QRS complex was divided into two windows, one leading and one trailing. When the leading window has a slope 3 times greater than the trailing window, that point was considered the onset of the QRS complex.

\subsection{Signal Averaging}

The HR bipole signal was enhanced via signal averaging to determine if His-Purkinje activity was observable. Because His-Purkinje activity is very low amplitude compared to other cardiac sources, the SNR needs to improve before His-Purkinje activity can be observed in the HR bipole lead.

To select beats to include in signal averaging, a template beat was selected from the dataset. The correlation with each beat and this template was taken, those beats with a $>0.9$ correlation were plotted against the template beat for visual inspection. Those passing visual inspection were included in signal averaging. To verify that no outliers were included each beat was plotted in an overlaying fashion. Figure 2 is an example of an overlaying display of the individual recordings from the HR bipole for one patient. This figure shows how well the large amplitude signals are aligned. All of the beats included in the average have the same characteristics (amplitude, shape, timing).

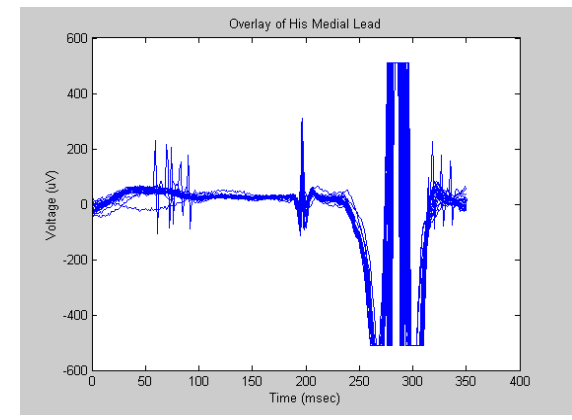

Figure 2. Overlay plots of all beats included in signal averaging of the $\mathrm{HR}$ bipole for one patient.

\section{3. $\quad$ Filtering}

A $4^{\text {th }}$ order Butterworth filter was applied to the summed data. The lower bound was tested from $10 \mathrm{~Hz}-$ $100 \mathrm{~Hz}$. Through analysis shown in Figure 3, the HisPurkinje activity was determined to best reveal itself with filters set at 30 and $200 \mathrm{~Hz}$.

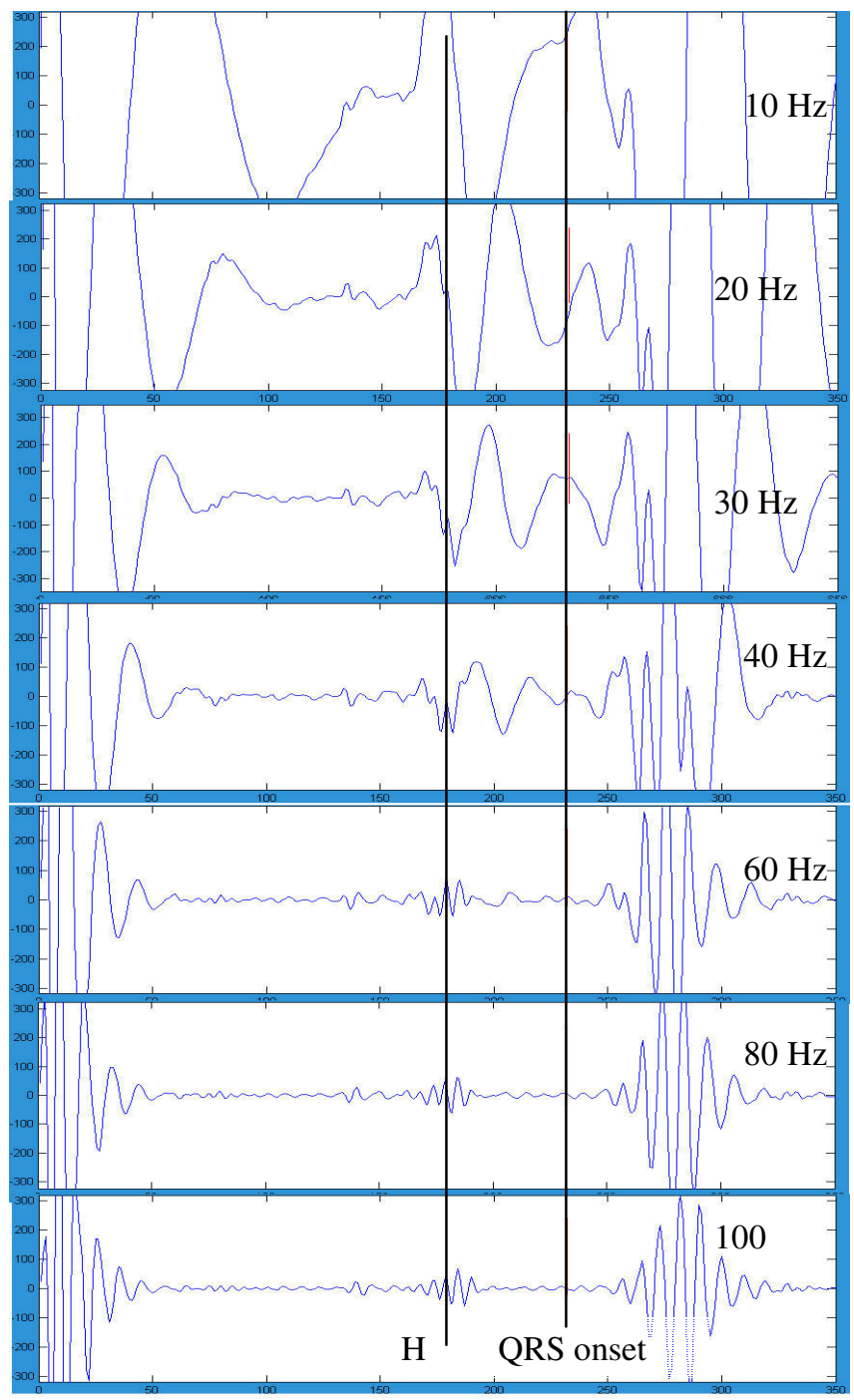

Figure 3: HR bipole signal filtered with $4^{\text {th }}$ order Butterworth bandpass filter. The frequency denoted on the right hand side of each plot is the lower bound. All plots have $200 \mathrm{~Hz}$ as the upper bound.

\subsection{Display for Analysis}

A GUI was developed to display the surface vector, HR bipole, and the distal HBE for comparison. All three plots have the onset of the QRS marked, either by an $*$ on the surface vector or a straight vertical line on the other two plots. This GUI also allowed for manipulation of voltage range to allow all aspects of the electrograms to be viewed. Figure 4 is a snapshot of the GUI displaying the surface vector, HR bipole (averaged) and the HBE (distal). 


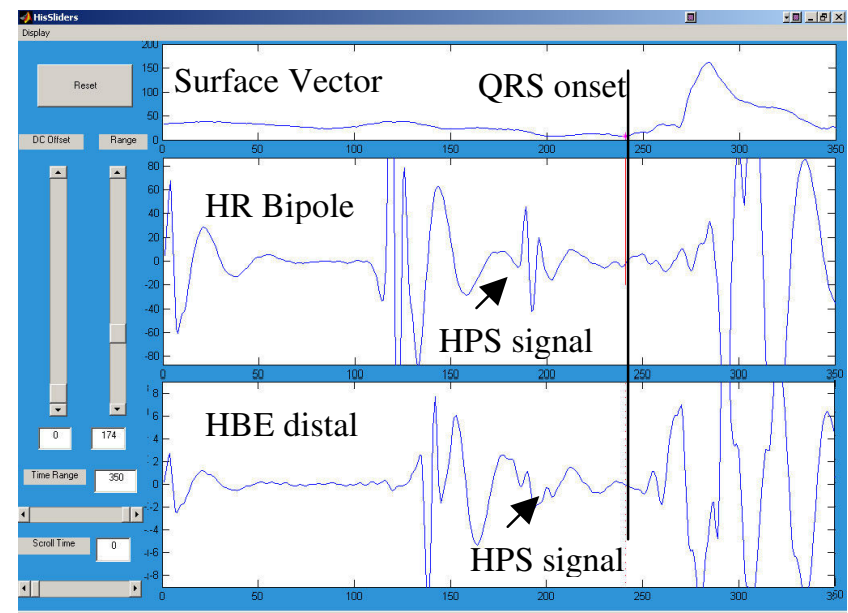

Figure 4: GUI developed to display signals for interpretation. To the left of the three plots are slides to adjust the range of time (horizontal axis) and voltage (vertical axis) shown.

\section{Results}

The best example of His Purkinje activity present in the HR bipole came during an atrial pacing dataset and is displayed in Figure 4. This is due to the very stable data that was captured during this time. Twenty-nine beats are included in this average. In Figure 4 the HR bipole provides a clear portion of His Purkinje activity from 190-240 milliseconds. The HBE provides verification of the initial His Bundle activity around 200 milliseconds. The onset of His Purkinje activity is visible due to atrial pacing and the extension of $\mathrm{A}-\mathrm{H}$ time.

\subsection{Testing Reproducibility}

Two subsets of data were taken as alternating beats from the same dataset. The HPS activity begins at the same point in the PR interval as where the HR bipole lead diverges from level (note arrows). The onset of HPS activity marked with arrows in Figure 5 is determined by tracing backwards through the PR interval starting at the onset of the QRS (vertical line). Tracing through the two sets of data should produce identical output as long as the signal is created by HPS activity. At the point where the two traces diverge is the point where HPS activity ends.

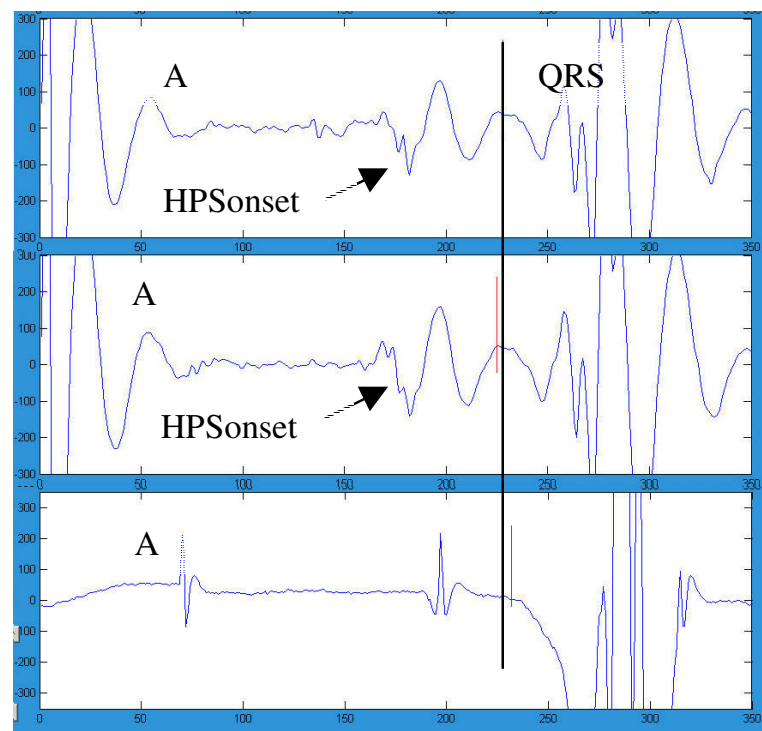

Figure 5: Using reproducibility to determine the onset of the HPS activity.

\subsection{Measuring HV Time}

Accuracy of the HV time measured in the HR bipole is judged by comparing these values to the HV time measured in the HBE. Figure 6 provides a linear regression to determine how well the HR bipole measurements matched with the accepted standard for these measurements, the HBE. The $r^{2}$ value of these data is calculated with Pearson's Product Moment coefficient to be 0.992 . This calculation was used to determine how well the data fit the linear model. A value of 0.992 means that $99.2 \%$ of variance among these data is accounted for by this linear fit. The offset of 18 milliseconds between the two recordings indicates a difference in where the HR bipole and the HBE begin to detect HPS activity.

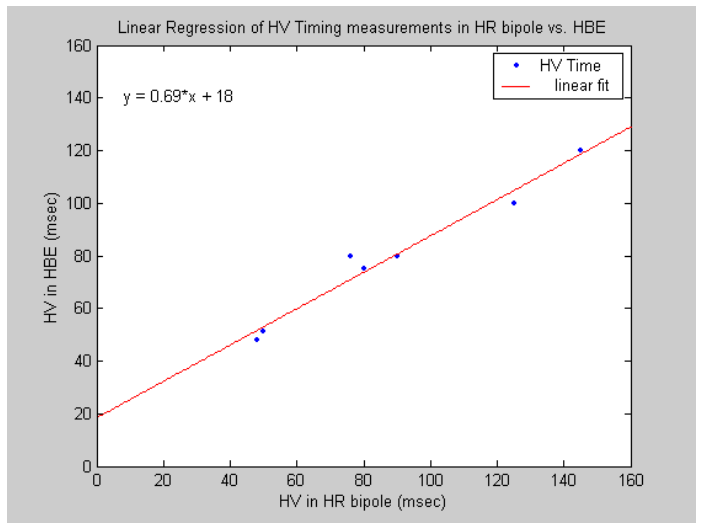

Figure 6: Linear regression of the relationship between HV time measurements from HR bipole and from HBE. 


\subsection{Reduced Resolution}

Taking the high-resolution signal and reducing it to the resolution of the current implantable device resolution was done to see if the HPS activity was still visible. A/D resolution was degraded from 12 bits to 8 bits and sampling rate reduced from $979 \mathrm{kHz}$ to 244.75 $\mathrm{Hz}$. The HPS activity in the HR bipole in Figure 7 is not visible; the position where it should be is marked with the arrow.

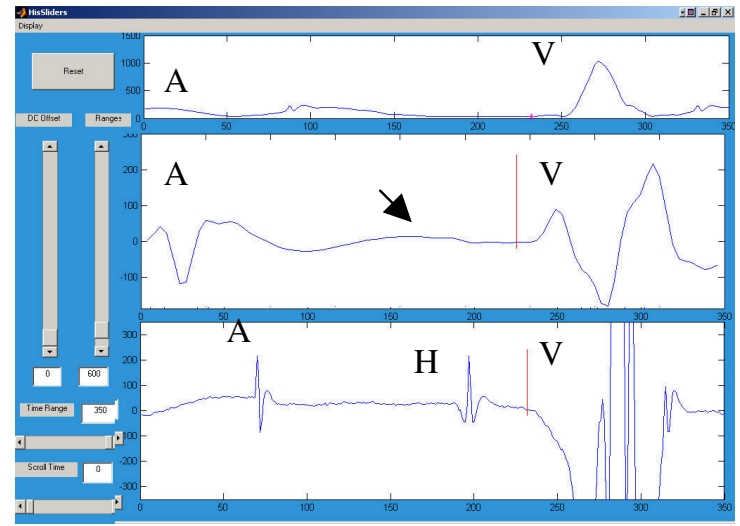

Figure 7: Data from Figure 5 with reduced resolution to 8 bit A/D and $244 \mathrm{~Hz}$ sampling rate. Arrow points to where HPS activity should be.

\section{Discussion and conclusions}

This study aimed to determine the feasibility of detecting HPS activity from the HR bipole. To test the success of detecting HPS activity, the HV interval was measured in both the HR bipole and the corresponding HBE. The number of datasets where this successfully occurred was only 7 out of a total of 38 datasets collected. While this value appears to say that detection of HPS activity in the HR bipole is not feasible, correcting a few of the obstacles should significantly improve the success rate.

In the EP studies, the catheters were not attached to the heart (they were "free-floating") and this proved to be a significant problem. The constant movement meant that even during the shortest set of data, the recording would not be consistent in amplitude and waveform to comply with the requirements of signal averaging. If it were possible to fix the electrodes in place (at least for the HRA and RVA to create a consistent HR bipole) then the number of beats included in the average would increase. Including more beats in the average would improve the SNR and better differentiate HPS activity from noise in the PR interval.

The measurement of the HV interval in the cases where HPS activity was visible in the HR bipole proved to be very consistent with the HV interval in the HBE. The linear relationship between the HV interval measured in the HR bipole and the HV interval measured in the HBE was ideal $\left(\mathrm{r}^{2}\right.$ of 0.992$)$ with the caveat of an $18 \mathrm{msec}$ offset due to the differences in detection of the onset of HPS activity. The consistency of HV measurements provides verification of the HR bipole in detection of HPS activity.

Alignment of signals for averaging was done with the QRS complex because this structure is the largest signal in the electrogram and typically remains consistent in duration in healthy patients. Alignment of the QRS leads to alignment of HPS signal. The AV time is not typically consistent. This is seen in Figure 2 as the $\mathrm{H}$ and $\mathrm{V}$ portions of the electrogram are aligned very well, the A portion can occur over a $50 \mathrm{msec}$ range of time, with no predominant time of occurrence. This atrial variability presents a problem in signal averaging (see Figures 2) because there is not a great deal of alignment to reinforce one location in the electrogram. The $\mathrm{H}$ and $\mathrm{V}$ are accurate in the signal-averaged plots because all of the beats present the same $\mathrm{H}$ and $\mathrm{V}$ waveforms occurring at the same point in each electrogram.

At reduced resolution, as shown in Figure 7, HPS activity is not visible. While the basic form of the electrogram in the HR bipole is maintained (atrial and ventricular activity are still visible and labeled A and V), the HPS activity appears lost in the PR interval. At what resolution the HPS activity will appear is something yet to be tested. While HPS activity was not clearly seen in this reduced form, further work could produce an algorithm that could find a trend in the area where the HPS activity should be.

Though the results of this feasibility study are not conclusive, they do appear promising and move a step closer to detecting HPS activity in pacemaker technology.

\section{Acknowledgements}

This research was funded by Medtronic, Inc.

\section{References}

[1] Berbari EJ, Steinberg JS. A Practical Guide to the Use of the High-Resolution Electrocardiogram. Armonk, NY: Futura Publishing Company, Inc., 2000.

Address for correspondence:

Edward J. Berbari, Ph.D.

Department of Biomedical Engineering, SL-174

Indiana University Purdue University Indianapolis

723 West Michigan Street

Indianapolis, IN 46202

eberbari@iupui.edu 